\title{
Metal Nitrides for Plasmonic Applications
}

Naik, Gururaj V.; Schroeder, Jeremy; Guler, Urcan; Ni, Xingjie; Kildishev, Alexander V.; Sands, Timothy D.; Boltasseva, Alexandra

\author{
Published in: \\ CLEO Technical Digest
}

Publication date:

2012

Document Version

Publisher's PDF, also known as Version of record

Link back to DTU Orbit

Citation (APA):

Naik, G. V., Schroeder, J., Guler, U., Ni, X., Kildishev, A. V., Sands, T. D., \& Boltasseva, A. (2012). Metal Nitrides for Plasmonic Applications. In CLEO Technical Digest (pp. QW3H.4). Optical Society of America.

\section{General rights}

Copyright and moral rights for the publications made accessible in the public portal are retained by the authors and/or other copyright owners and it is a condition of accessing publications that users recognise and abide by the legal requirements associated with these rights.

- Users may download and print one copy of any publication from the public portal for the purpose of private study or research.

- You may not further distribute the material or use it for any profit-making activity or commercial gain

- You may freely distribute the URL identifying the publication in the public portal

If you believe that this document breaches copyright please contact us providing details, and we will remove access to the work immediately and investigate your claim. 


\title{
Metal Nitrides for Plasmonic Applications
}

\author{
Gururaj V. Naik ${ }^{1}$, Jeremy Schroeder ${ }^{2}$, Urcan Guler ${ }^{1}$, Xingjie Ni ${ }^{1}$, Alexander V. Kildishev ${ }^{1}$, Timothy D. \\ Sands $^{2}$ and Alexandra Boltasseva*,1,3,4 \\ ${ }^{I}$ School of Electrical and Computer Engineering and Birck Nanotechnology Center, Purdue University, West Lafayette, IN 47907, USA \\ ${ }^{2}$ School of Materials Engineering and Birck Nanotechnology Center, Purdue University, West Lafayette, IN 47907, USA \\ ${ }^{3}$ DTU Fotonik, Department of Photonics Engineering, Technical University of Denmark, Lyngby, DK-2800, Denmark \\ ${ }^{4}$ Erlangen Graduate School of Advanced Optical Technologies (SAOT), Universität Erlangen-Nürnberg, 91052 Erlangen, Germany \\ Email: aeb@purdue.edu*
}

\begin{abstract}
Metal nitrides as alternatives to metals such as gold could offer many advantages when used as plasmonic material. We show that transition metal nitrides can replace metals providing equally good optical performance for many plasmonic applications

OCIS codes: (160.4670) Optical materials; (250.5403) Plasmonics; (160.3918) Metamaterials;
\end{abstract}

Plasmonics and metamaterials (MMs) have provided a new dimension to the scope and applications of nano-optics [1]. However, there are many practical difficulties associated with realization of the proposed MM devices that hinder their real-life applications. One of the major problems in the optical range is loss associated with the plasmonic constituents of MMs [2]. Another critical factor is that the real part of dielectric permittivity in conventional metals is too large in magnitude to be readily suitable for many MM designs [3]. Thus, alternative plasmonic materials are essential for many MM devices operating in the optical range. In the search for better plasmonic materials, transparent conducting oxides (TCOs) were proposed as low loss alternatives to gold and silver in the near-infrared [4]. In this paper, we study metal nitrides as potential candidates for alternative plasmonic materials at visible wavelengths. We provide performance estimates for plasmonic devices with titanium nitride as an alternative to metal and demonstrate plasmonic property of titanium nitride by exciting surface plasmon polaritons (SPPs) using a grating coupler.

Transition metal nitrides such as $\mathrm{ZrN}$, TiN, TaN and $\mathrm{HfN}$ exhibit plasmonic property (negative real part of permittivity) when deposited as metal-rich films. The optical properties of these materials deposited as thin films using DC reactive sputtering are shown in Fig. 1a and 1b. For comparison the figures plot dielectric functions of gold and silver. The real permittivity of nitrides is much smaller in magnitude than that of $\mathrm{Au}$ or $\mathrm{Ag}$ in the visible range while the losses are comparable to that of gold for $\mathrm{ZrN}$ and $\mathrm{TiN}$ films and higher for all other nitrides. This makes $\mathrm{Ti}$ - and $\mathrm{Zr}$ - nitrides preferable for many plasmonic and $\mathrm{MM}$ applications. One example is an important class of MMs called hyperbolic metamaterials (HMMs) [5]. They can be formed by stacking sub-wavelength thick, alternating layers of metallic and dielectric materials. The figure-of-merit (FoM) of such HMMs is given by $\beta^{\prime} / \beta^{\prime \prime}$, where $\beta$ is the propagation vector of the waves propagating in the direction perpendicular to the layers [6]. The FoM for many different metal/dielectric systems are plotted in Fig. 1c. Clearly, HMMs that use metal nitrides instead of metals out-perform gold- or silver-based HMMs by 3 orders of magnitude in the visible range. Thus, metal nitrides are better alternatives to gold and silver in the visible frequencies for HMM applications. For plasmonic applications such as sensors, the field-enhancement produced by localized surface plasmon resonance (LSPR) in metal nanoparticles plays a central role. Conventionally gold nanoparticles were used in such applications providing intensity enhancement of about 35 around the wavelength of $550 \mathrm{~nm}$ [7]. TiN nanoparticles when used instead of gold for such applications could provide comparable field enhancement (see Fig. 1d). In the wavelength range from $750 \mathrm{~nm}$ and longer TiN provides higher field enhancement than gold nanoparticles thus being material of preference in this spectral range.

Titanium nitride is a low-loss material with plasmonic properties in the visible frequencies. To demonstrate plasmonic behavior we show that SPPs can be excited at the TiN/dielectric interface in the near-infrared range. In order to couple SPPs onto TiN thin film dielectric gratings are made on top of the TiN film. The gratings are formed by patterning about $100 \mathrm{~nm}$ thick e-beam resist (ZEP) spin-coated on top of the TiN film with a periodicity of 1.5 $\mu \mathrm{m}$. The angular reflectance of this structure was measured at wavelengths of 900 and $1000 \mathrm{~nm}$ (see Fig. 1e). The dip in the reflectance at 32 and 26 degrees respectively correspond to the SPP excitation in the TiN/ZEP interface. This was confirmed by performing simulations based on spatial-harmonic-analysis (SHA) [8]. The calculations match reasonably well with the measured curves confirming the excitation of SPPs on the surface of the TiN thin film. 
a)

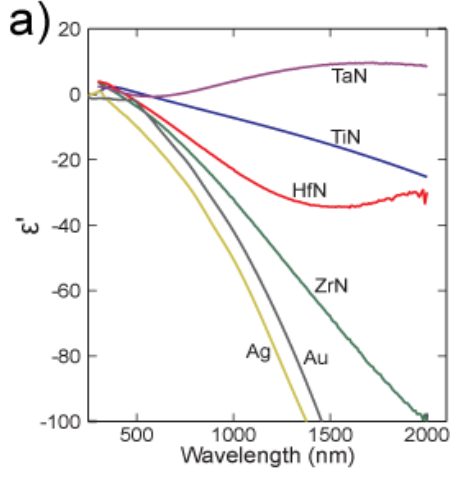

d)

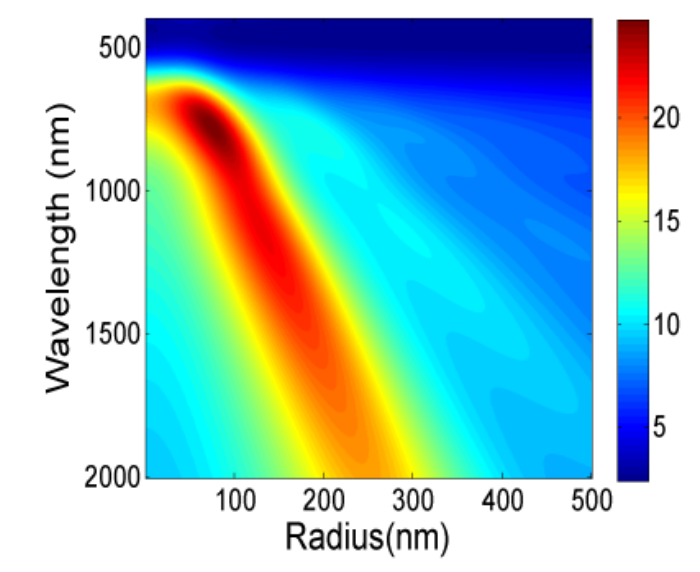

b)

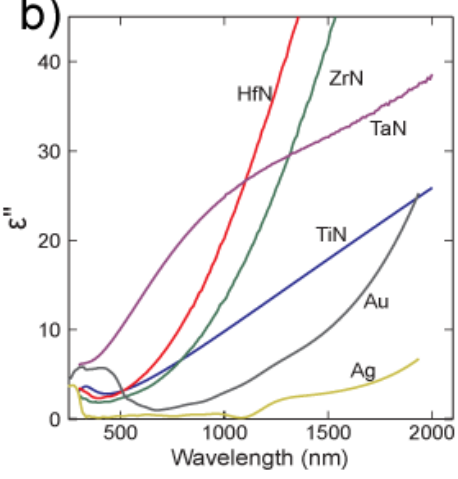

c)

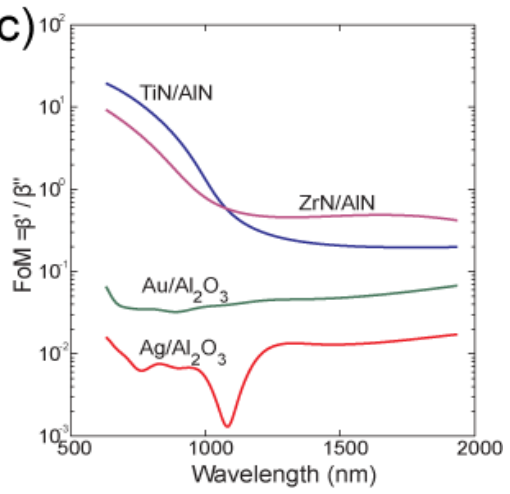

e)

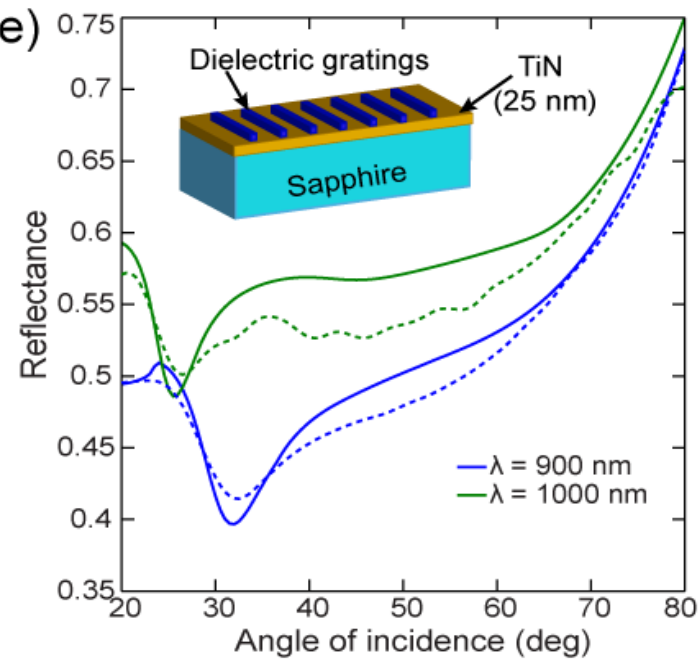

Fig 1. Optical properties of transition metal nitrides: a) real and b) imaginary parts of permittivity of TiN, TaN, HfN and ZrN films extracted from ellipsometry measurements (specify the machine). The dielectric function of gold and silver are from ref.[9]. c) Figure-of-merit of planar hyperbolic metamaterial (HMM) made using different material systems. d) The intensity enhancement in a spherical nanoparticle of TiN calculated from Mie theory plotted for different radii of the sphere. e) The oblique incidence reflectance measurement from TiN film with dielectric (ZEP polymer) gratings on top (inset shows the schematic of the sample). The measured results are in solid lines and the dotted lines are calculated curves based spatial-harmonic-analysis (SHA).

In conclusion, metal nitrides can outperform gold in the visible and near-infrared spectra for specific MM and plasmonic applications. As the first step towards plasmonic devices utilizing these new materials, we demonstrate excitation of SPPs on TiN thin film. Metal nitrides as alternative plasmonic materials in the visible and near-infrared spectra could overcome many disadvantages associated with conventional metals.

\section{References}

[1] J. B. Pendry, et al., "Controlling Electromagnetic Fields," Science, vol. 312, pp. 1780-1782, June 23, 20062006.

[2] A. Boltasseva and H. A. Atwater, "Low-loss plasmonic metamaterials," Science, vol. 331, p. 290, 2011.

[3] A. Kildishev and V. Shalaev, "Engineering space for light via transformation optics," Optics letters, vol. 33, pp. 43-45, 2008.

[4] G. V. Naik, et al., "Oxides and nitrides as alternative plasmonic materials in the optical range [Invited]," Opt. Mater. Express, vol. 1, pp. 1090-1099, 2011.

[5] Z. Jacob, et al., "Optical Hyperlens: Far-field imaging beyond the diffraction limit," Opt. Express, vol. 14, pp. 8247-8256, 2006.

[6] A. Hoffman, et al., "Negative refraction in semiconductor metamaterials," Nature Materials, vol. 6, pp. 946-950, 2007.

[7] K. L. Kelly, et al., "The optical properties of metal nanoparticles: the influence of size, shape, and dielectric environment," The Journal of Physical Chemistry B, vol. 107, pp. 668-677, 2003.

[8] X. Ni, et al., "The validation of the parallel three-dimensional solver for analysis of optical plasmonic bi-periodic multilayer nanostructures," Applied Physics A: Materials Science \& Processing, vol. 100, pp. 365-374, 2010.

[9] P. B. Johnson and R. Christy, "Optical constants of the noble metals," Physical Review B, vol. 6, p. 4370, 1972. 